\title{
The performance of Space Time Block Coding (STBC) in MIMO relay network
}

\author{
Hamza Eldenferia ${ }^{1 *}$, Jamal Elbergali ${ }^{2}$ \\ 1,2 Electronic Engineering Department- College of Industrial Technology Misurat- Libya \\ DOI: https://doi.org/10.21467/proceedings.2.5 \\ * Corresponding author email: hamza.ham1985@gmail.com
}

\begin{abstract}
This paper is presenting the performance analysis of a cooperative MIMO(MultipleInput-Multiple-Output) relaying system with a single relay based on Alamouti scheme. It examines the Space Time Codes (STC) techniques where the focus is on the Space Time Block Coding (STBC). The MIMO system is built on Alamouti Space Time Block Coding (STBC) over Rayleigh flat fading channels. The source and destination nodes are equipped with two transmit antennas while the relay node is equipped with multiple antennas and Amplifiers-and-Forwards (AF). In addition the receiver uses the Zero Forcing $(\mathrm{ZF})$ equalizer. The system reliability will be evaluated by using of bit error rate (BER) performance.

Keywords: MIMO relay network; Alamouti scheme; Space Time Block Coding (STBC); Zero Forcing (ZF) equalizer; bit error rate (BER)
\end{abstract}

\section{Introduction}

Wireless communications have recently turned to a technique known as Multiple Input Multiple Output (MIMO) to improve the quality (bit-error rate) and data rate (bits/sec). MIMO technology has attracted attention in wireless communications, because it offers significant increases in data throughput and link range without additional bandwidth or increasing transmit power [1]. This is done by using multiple transmit and receive antennas, as well as suitable coding techniques. They take benefit of spatial and temporal diversity to combat the random fading induced by multi-path propagation of the signal and maximize efficient use of bandwidth. There is also a fundamental gain in transmitting data over a matrix rather than vector channel. Transmission of data over MIMO channels has traditionally focused on data rate maximization or diversity maximization [2]. Recently, Cooperative communication [3] has attracted a lot of attention because of its ability to enhance the system performance. Cooperative communication concerns a system where users share and organize their resources to improve the transmission quality and enhance the power allocation. The combination of MIMO processing with cooperative relaying helps to improve the capacity of the overall cooperative system [4]. In recent times, it has been revealed that cooperation based on Space-Time Block Codes (STBC) gives an effective method to present spatial diversity in various wireless scenarios [5][6].

\subsection{Multi - Antenna Transmission Methods}

To transmit information over a single wireless link, different transmission and reception strategies can be applied. Which one of them should be used depends on the knowledge of the instantaneous MIMO

(C) 2018 Copyright held by the author(s). Published by AIJR Publisher in Proceedings of First Conference for Engineering

Sciences and Technology (CEST-2018), September 25-27, 2018, vol. 1.
This is an open access article under Creative Commons Attribution-NonCommercial 4.0 International (CC BY-NC 4.0)

A iR license, which permits any non-commercial use, distribution, adaptation, and reproduction in any medium, as long as the original work is properly cited. ISBN: 978-81-936820-5-0 
channel parameters at the transmitter side. If the Channel State Information (CSI) is not available at the transmitter, Spatial Multiplexing (SM) or Space-Time Coding (STC) can be used for transmission. If the CSI is available at the transmitter, beamforming can be used to transmit a single data stream over the wireless link. In this way, spectral efficiency and robustness of the system can be improved [1]. It can be concluded that the choice of the transmission model depends on three entities important for wireless link design, namely bit rate, system complexity and reliability. STC has low complexity and promises high diversity, but the bit rate is moderate. SM provides high bit rate, but is less reliable. Beamforming exploits array gain, is robust with respect to channel fading, but it requires CSI at both transmitter and receiver. In this research only STC transmission has been considered.

Let us consider a point-to-point MIMO system with $n_{t}$ transmit and $n_{r}$ receive antennas. The block diagram is given in Figure 1. Let $h_{i, j}$ be a complex number corresponding to the channel gain between transmit antenna $j$ and receive antenna $i$ [2][3].

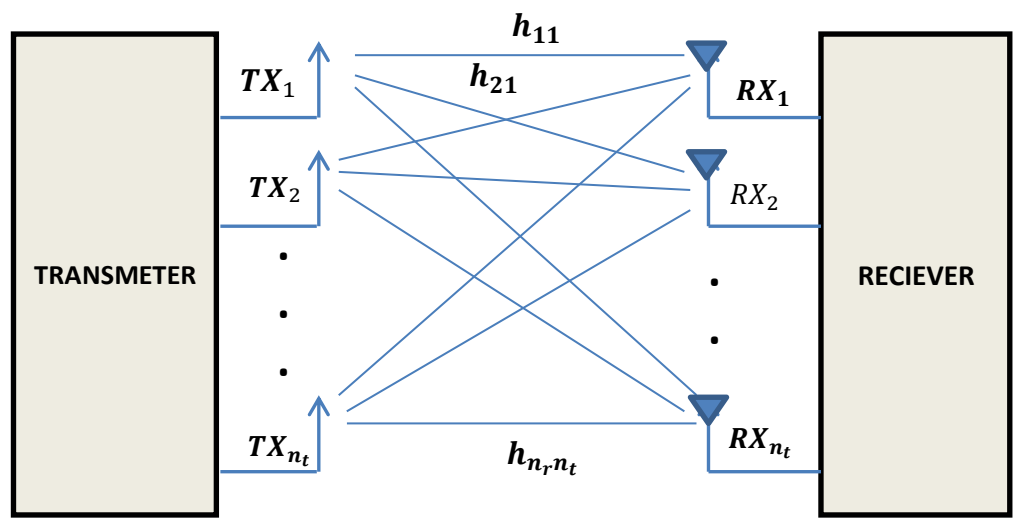

Figure 1. MIMO model with $n_{t}$ transmit antennas and $n_{r}$ receive antennas.

If at a certain time instant the complex signals $\left\{x_{1}, x_{2}, \cdots, x_{n_{t}}\right\}$ are transmitted via $n_{t}$ transmit antennas, the received signal at antenna $i$ can be expressed as [7]:

$$
y_{i}=\sum_{j=1}^{n_{t}} h_{i, j} x_{j}+n_{i}
$$

Where $n_{i}$ is a noise term. Combining all receive signals in a vector $Y$, this equation can be easily expressed in matrix form [7]:

$$
Y=H x+n
$$

$Y$ is the $n_{r} \times 1$ receive symbol vector, $H$ is the $n_{r} \times n_{t}$ MIMO channel transfer matrix, $x$ is the $n_{t} \times 1$ transmit symbol vector and $n$ is the $n_{r} \times 1$ additive noise vector.

\subsection{Wireless Relaying Systems}

The main advantages of using relay are to increase coverage and decrease the need to use high power at the transmitter [8][9][10]. Essentially, in relaying systems the source terminal uses other terminals or relays to forward its information to the destination terminal. Generally, there are two types of relaying 
The performance of Space Time Block Coding (STBC) in MIMO relay network

systems, Decode-and-Forward (DF) relaying systems, and Amplify-and-Forward (AF) systems. In this paper AF system is used to implement the Model. Recently, with a good understanding of the benefits of MIMO systems, scientists have suggested some methods that could help to achieve the advantages of both MIMO and wireless relaying systems.

\section{System Model}

In this paper, we present performance analysis of a MIMO relaying system based on Alamouti scheme. As shown in Figure 2, the system considered two antennas at source and destination and one relay centred exactly between the source and destination. BPSK signal modulation is used to generate equal probability of ' 0 's and ' 1 's at the transmitter side.

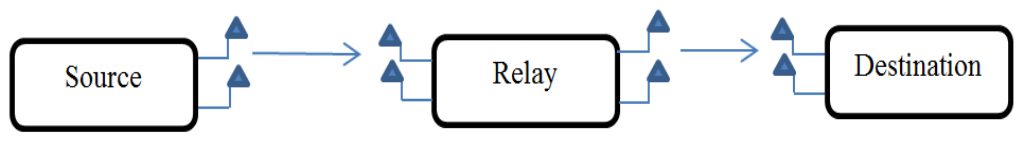

Figure 2. $2 \times 2$ MIMO relaying system.

At the relay node, we have used AF algorithm. The system channel between source, relay and relay destination are simply used as Rayleigh flat-fading channel with Additive White Gaussian Noise (AWGN) with zero mean. Also the Zero Forcing (ZF) technique has been used as equalizer at the receiver side.

\subsection{Space Time Block Coding scheme}

In this paper, two transmit and receive antennas is used. The input information stream $\boldsymbol{x}(t)$ is encoded by STBC encoder. Alamouti suggested that group the symbols into groups of two. In the first time slot, send $\boldsymbol{x}_{1}$ and $\boldsymbol{x}_{2}$ from the first and second antenna. In second time slot send $\boldsymbol{x}_{1}^{*}$ and $-\boldsymbol{x}_{2}^{*}$ from the first and second antenna. Where $(.)^{*}$ denoted to complex conjugate function. Thus, at two time slots the input symbols are given by [10]:

$$
x(t)=\left(\begin{array}{cc}
x_{1} & -x_{2}^{*} \\
x_{2} & x_{1}^{*}
\end{array}\right)
$$

The encoded information is transmitted through Rayleigh fading channel and AWGN as addition noise. The received signal vector at the receiver can be given like:

In the first time slot, the received signal is [7]:

$$
\left(\begin{array}{l}
y_{1}^{1} \\
y_{2}^{1}
\end{array}\right)=\left(\begin{array}{ll}
h_{11} & h_{12} \\
h_{21} & h_{22}
\end{array}\right)\left(\begin{array}{l}
x_{1} \\
x_{2}
\end{array}\right)+\left(\begin{array}{l}
n_{1}^{1} \\
n_{2}^{1}
\end{array}\right)
$$

In the second time slot, the received signal is:

$$
\left(\begin{array}{l}
y_{1}^{2} \\
y_{2}^{2}
\end{array}\right)=\left(\begin{array}{ll}
h_{11} & h_{12} \\
h_{21} & h_{22}
\end{array}\right)\left(\begin{array}{c}
-x_{2}^{*} \\
x_{1}^{*}
\end{array}\right)+\left(\begin{array}{l}
n_{1}^{2} \\
n_{2}^{2}
\end{array}\right)
$$

Combining the equations at time slot 1 and 2 . 


$$
\left(\begin{array}{c}
y_{1}^{1} \\
y_{2}^{1} \\
y_{1}^{2^{*}} \\
y_{2}^{2^{*}}
\end{array}\right)=\left(\begin{array}{cc}
h_{11} & h_{12} \\
h_{21} & h_{22} \\
h_{12}^{*} & -h_{11}^{*} \\
h_{22}^{*} & -h_{21}^{*}
\end{array}\right)\left(\begin{array}{l}
x_{1} \\
x_{2}
\end{array}\right)+\left(\begin{array}{c}
n_{1}^{1} \\
n_{2}^{1} \\
n_{1}^{2^{*}} \\
n_{2}^{2^{*}}
\end{array}\right)
$$

\subsection{Relay Procedure}

The received signal $y_{r}$ at relay node is amplified by matrix $\boldsymbol{F}$ and retransmitted to the destination node. The relaying matrix $\boldsymbol{F}$ can be expressed by [10][11][12]:

$$
F=\beta_{r} I_{N_{r}}
$$

Where $\beta_{r}$ is the amplifying factor of the relay and $\boldsymbol{I}_{N_{r}}$ is the $N_{r} \times N_{r}$ identity matrix. The amplifying factor is calculated by [11][12]:

$$
P_{a}=\beta_{r}{ }^{2} \operatorname{tr}\left\{F\left(H_{r} H_{r}^{H}+I_{N_{r}}\right) F^{H}\right\}
$$

Where $P_{a}$ is the transmit power in relay node, $(.)^{H}$ represent the Hermitian complex conjugate transpose and $\operatorname{tr}($.$) denotes to trace of a matrix[9][8].$

\subsection{Zero Forcing (ZF) Algorithm}

The ZF equalizer is used in receivers to alleviate the effects of ISI. Therefore, to reach reasonable system performance and to decrease the complexity of the equalizer, ZF equalization technique has been used in this research [7]. The equivalent channel matrix with noise given by [11][12]:

$$
\begin{aligned}
& H=H_{r, d} F H_{r} \\
& n=H_{r, d} F n_{r}+n_{r, d}
\end{aligned}
$$

Where $H_{r}$ is the channel between source and relay and $H_{r, d}$ is the channel between relay and destination.

The estimated received signal $\hat{x}$ can be determined as following:

$$
\hat{x}=W_{Z F} Y
$$

Where $W_{Z F}=H\left(H^{H} H\right)^{-1}$, is known as the ZF pseudo-inverse for a general $m \times n$ matrix and $(.)^{-1}$ indicates simple matrix inversion.

\section{Results and Discussion}

In this research two-hops MIMO relay network with STBC is simulated by MATLAB software. The MATLAB program used to highlight the performance of STBC MIMO relay compared with STBC MIMO without using relay (Direct connection). In addition, we have examined the optimum location of the relay, by fixing the Signal to Noise Ratio (SNR) at $20 \mathrm{~dB}$ and make the distance varied.

In this program the BPSK modulation is used to modulate the information, and also STBC encoder and decoder is used at the source and destination sides. The backward channel (source to relay) and the forward channel (relay to destination) are Rayleigh fading channel with zero mean complex circular AWGN. In addition, the ZF relay scheme is used at the receiver side as equalizer to alleviate the effects of ISI. At the relay node the AF technique has been used because it's easy to implement and the results near to practice one. The results have been divided to two sections:Section I: The performance is 
The performance of Space Time Block Coding (STBC) in MIMO relay network

measured by means of calculating the Bit Error Rate (BER) and plotting against the SNR between the source and destination, where the SNR is varied between 0 to $30 \mathrm{~dB}$. The MIMO network is used, with and without using relay. The distance in this section has been fixed as D for source-relay and relay destination, and 2D for direct connection (without aid of relay) as shown in Figure 3.

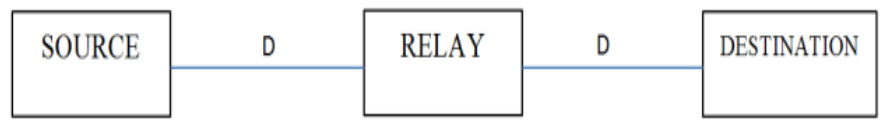

\begin{tabular}{|l|l|l|}
\hline SOURCE & 20 & DESTINATION \\
\cline { 3 - 3 } & & \\
\hline
\end{tabular}

Figure 3. The distance considerations.

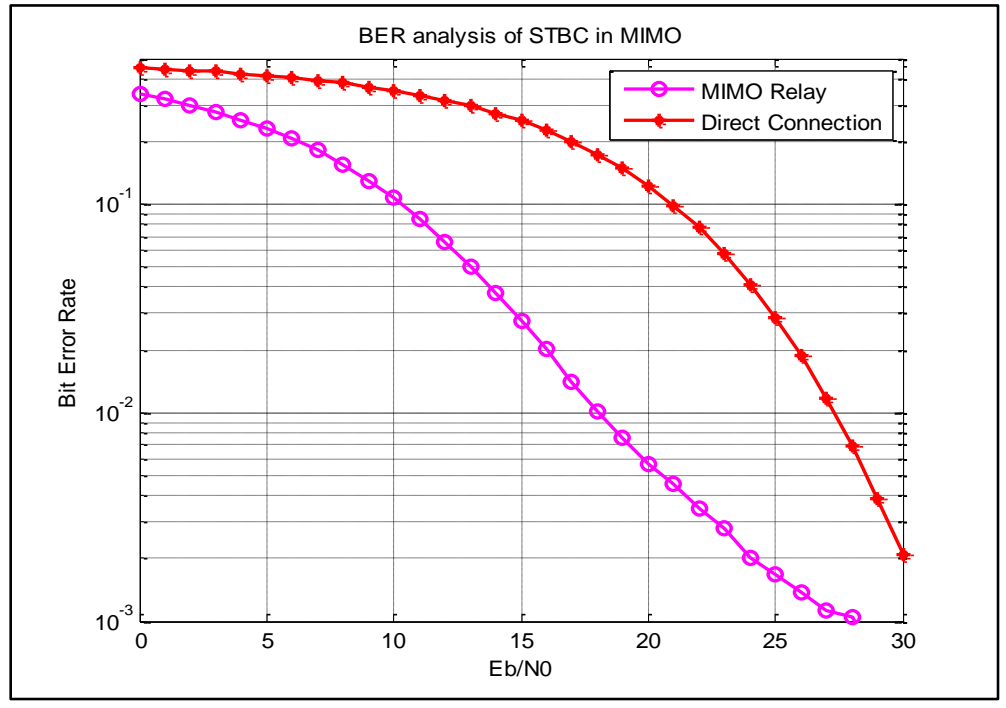

Figure 4. BER for MIMO using relay (MIMO relay) and MIMO without relay (Direct Connection).

As can be seen from Figure 4, the curves show the performance of MIMO using relay (MIMO relay) and MIMO without using relay (Direct Connection) between the source and destination using $\mathrm{ZF}$ algorithm for both. It can be observed that the BER performance for (MIMO relay) network is much better than the performance of MIMO (Direct Connection) network that's because the relay amplify and forward the information at distance D and retransmitted it again while in direct connection the source transmit the information directly over distance 2D with the same transmitted power. So, when we compare the BER plot, we can see that (Direct Connection) network has around $3 \mathrm{~dB}$ poorer performance than (MIMO relay) network. That's introducing the relay network in the MIMO system minimize the BER which is more constructive in practical communication systems.

Section II: In this section the SNR has been fixed at $20 \mathrm{~dB}$ and the distance between the source and relay is varied. This step has been taken to find out the optimum location for the relay between the 
source and destination. In this part, we estimate the distance between the source and destination as unit function equal to 1 , so the distance between source to relay is $\mathrm{D}$ and relay destination $\mathrm{B}=(1-\mathrm{D})$. Therefore, the relay location starts at 0.1 and ends at 0.9 .

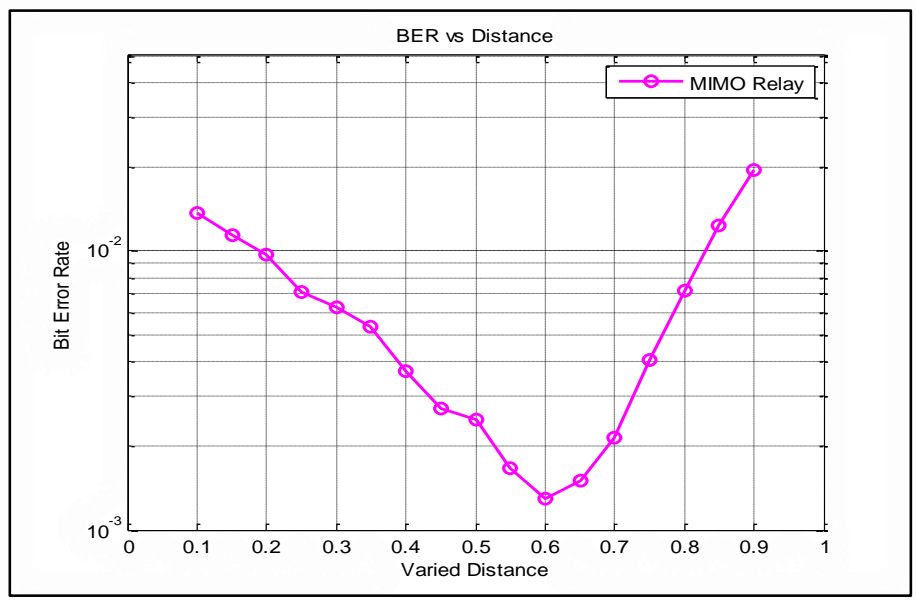

Figure 5. Find out the optimum location of the relay between the source and destination.

As can be seen from Figure 5 the curve shows optimum location of the relay between source and destination and the effects of changing the relay location. The curve shows the best location for the relay between source and destination. As can be seen from output performance the best place for relay is almost in the middle distance between source and destination. In addition, the worst case when the relay was near to the destination.

\section{Conclusion}

In conclusion, the aim of this paper was the performance of Space Time Block Codes (STBC) in MIMO relay communication systems. The research showed the relevant theories for MIMO relay network and STBC coding in order to get the appreciation for the theoretical predictions of the performance of the MIMO relay network systems. The simulation used the STBC based on multi-antenna cooperative systems and provided the corresponding simulated performance under Rayleigh fading channel AF algorithm and ZF equalizer. The system performance is improved more with the (MIMO relay) network than the MIMO system without the aid of relay network (Direct connection). In addition, to get the best performance of the MIMO relay network the relay has to be at the middle distance between the sources and destination.

\section{References}

[1] M. Alamouti, “A Simple Transmit Diversity Technique for wireless communications", IEEE Journal on sele areas in communications, Vol. 16, No. 8, October 1998.

[2] D.Gesbert, M.Shafi, D.S.Shiu, P.Smith and A.Naguib, " From Theory to Practice: An Overview of MIMO spacetime coded wireless systems".

[3] A. Stefanov, E. Erkip, On the performance analysis of cooperative space-time coded systems, in: IEEE Wireless Communications and Networking, 16-20 March 2003, vol. 2, pp. 729-734. 
The performance of Space Time Block Coding (STBC) in MIMO relay network

[4] Y.Wang, F.Liu, S. Xu, X.Wang, Y. Qian and P.Wang, "Performance Analysis of Multi-hop MIMO Relay Network", IEEE Communications society, ICC 2008 workshop proceedings.

[5] A.Nosratinia, E.Hunter and A.Hedayat, "Cooperative Communications in Wireless Networks", Adaptive Antennas and MIMO Systems for Wireless Communications, IEEE Communications Magazine, October 2004.

[6] J. N. Laneman, D. Tse, and G. W. Wornell, "Cooperative diversity in wireless networks: Efficient protocol and outage behavior," IEEE Trans. on Information Theory, vol. 50, no. 12, pp. 3062-3080, Dec 2004.

[7] N.Ngajikin, W.N.Ahmad, N.Fisal and S.K.Yusof, "Simulation on Performance of Space time Block Code", $R F$ and Microwave Conference, October 2004.

[8] Y.fan and J.Thompson, "MIMO Configurations for Relay Channels: Theory and Practice", IEEE Transactions on wireless communications, Vol. 6, No. 5, May 2007,

[9] S.Behbanhani and R.Merched, M. Eltawil, "Optimizations of a MIMO Relay Network”, IEEE transactions on signal processing, Vol. 56, No. 10, October 2008,

[10] S. Atapattu and N. Rajatheva, "Exact SER of Alamouti code transmission through amplify-forward cooperative relay over Nakagami-m fading channels," in Proc. IEEE International Symposium on Communications and Information Technologies ISCIT '07, 2007, pp. 1429-1433.

[11] Y. Song, H. Shin, and E. Hong, "MIMO cooperative diversity with scalar-gain amplify-and-forward relaying," IEEE Trans. Commun.,vol. 57, no. 7, pp. 1932-1938, Jull 2009.

[12] Y. Ding, J. K. Zhang and K. M. Wong, "The Amplify and Forward Half Duplex Cooperative System: Pairwise Error Probability And Precoder Design”, IEEE Transaction on Signal Processing, Vol. 55, No. 2, pp. 605-617, February 2007. 